\title{
[gw22-e0119] ACUTE MYOCARDIAL ISCHAEMIA DIRECTLY MODULATES THE EXPRESSION OF BRAIN NATRIURETIC PEPTIDE AT THE TRANSCRIPTIONAL AND TRANSLATIONAL LEVELS VIA INFLAMMATORY CYTOKINES
}

Xia Wenjing, Huang Yiyi, Chen Yiyi, Chen Shenglong First Affiliated Hospital Of Sun Yat-sen University, Guangzhou, China

\subsection{6/heartjnl-2011-300867.343}

Cardiomyocyte stretching has been reported to be a major trigger for brain natriuretic peptide (BNP) release; however, an increase in circulating BNP is observed in patients with acute myocardial ischaemia in the absence of increased left ventricular wall stress. In the present study, to investigate the direct and independent effects of acute myocardial ischaemia on BNP expression and its mechanism, we established a ischaemia and hypoxia injured model of cultured rat cardiomyotes and proved hypoxia upregulated expressions of interleukin 6 and BNP. Further treatment with IL- 6 elicited dose- and time-dependent increases in BNP mRNA and protein expression as well as an upregulation in transforming growth factor $\beta 1$ (TGF $\beta 1$ )/Smad2 expression, which was partially suppressed by a neutralising antibody. In conclusion, our study showed that acute myocardial ischaemia can directly upregulate BNP expression at the translational and transcriptional levels through the action of IL- 6 , and this process is associated with the upregulation of TGF $\beta 1 / \mathrm{Smad} 2$ signaling. 